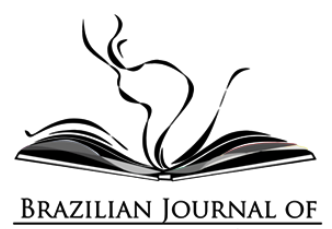

\title{
PANDEMIA, INEQUIDAD Y PROTECCIÓN SOCIAL NEOLIBERAL: CHILE, UN CASO PARADIGMÁTICO
}

\author{
PANDEMIA, DESIGUALDADE E PROTEÇÃO SOCIAL NEOLIBERAL: \\ CHILE, UM CASO PARADIGMÁTICO
}

PANDEMIC, INEQUITY AND NEOLIBERAL SOCIAL PROTECTION:

CHILE, A PARADIGMATIC CASE

Ana Farías Antognini

María Paz Trebilcock (iD)2

Universidad Alberto Hurtado, Chile

Resumen: El presente estudio tiene como objetivo analizar las políticas de protección social que se dispusieron en Chile para enfrentar la pandemia en el marco de una estructura de bienestar liberal. La protección social se ha visto fuertemente estresada por el impacto de la pandemia del COVID-19, haciendo frente a los graves problemas de desempleo y disminución de ingresos de las familias. El carácter de las medidas dispuestas para la emergencia depende de la estructura de bienestar que han construido los países, siendo Chile un caso paradigmático de régimen neoliberal. Las decisiones para combatir los impactos socioeconómicos han mantenido un carácter subsidiario con fuerte predominio del sector privado, con coberturas focalizadas donde la atención del Estado ha estado centrada especialmente en la población más vulnerable. Las características del reparto de las prestaciones sociales, no han logrado mayores niveles de equidad y cohesión social, sino que por el contrario alejan la posibilidad de proveer prestaciones de carácter universal al no incorporar componentes redistributivos y solidarios en la seguridad social. La continuidad en la estructura de bienestar neoliberal nos lleva a plantear que las políticas sociales, especialmente aquellas vinculadas a la protección social han visualizado y ensanchado las grandes brechas de inequidad en la población.

\footnotetext{
'Doctora en Ciencias Políticas. Universidad Católica de Chile, Académica Universidad Alberto Hurtado afarias@uahurtado.cl

${ }^{2}$ Doctora en Sociología. Universidad Católica de Chile, Investigadora Adjunta del Programa Iniciativa Milenio ICS2019_025 "Instituto Milenio para la Investigación en Violencia y Democracia, VIODEMOS, Académica de la Universidad Alberto Hurtado mtrebilcock@uahurtado.cl
}

Cadernos Prolam/USP-Brazilian Journal of Latin American Studies, v. 20, n. 40, p. 189-210, out. 2021

189 Edição especial: Trabalho e proteção social na América Latina: desenvolvimentos recentes e os desafios diante da crise pandêmica. ISSN: 1676-6288 
Palabras clave: COVID-19; Política Social, Protección Social; Neoliberalismo; Desigualdad.

Resumo: $O$ presente estudo tem como objetivo analisar as políticas de proteção social implementadas no Chile para enfrentar a pandemia no marco de uma estrutura liberal. A proteção social tem sido fortemente pressionada pelo impacto da pandemia COVID-19 em um cenário de graves problemas de desemprego e diminuição da renda familiar. A natureza das medidas previstas para a emergência depende da estrutura de bem-estar que os países construíram, sendo o Chile um caso paradigmático de regime neoliberal. As decisões de combate aos impactos socioeconômicos têm mantido um caráter subsidiário com forte predomínio do setor privado, com coberturas do Estado focadas principalmente na população mais vulnerável. As características da distribuição dos benefícios sociais não têm alcançado melhor nível de equidade e coesão social, pelo contrário, afastam a possibilidade de universalização dos benefícios ao não incorporar componentes redistributivos e solidários na seguridade social. A continuidade da estrutura neoliberal nos leva a argumentar que as políticas sociais, especialmente aquelas vinculadas à proteção social, visualizaram e expandiram as grandes lacunas de desigualdade da população.

Palavras-chave: COVID-19; Política Social, Proteção Social, Neoliberalismo; Desigualdade.

Abstract: The present study aims to analyze the social protection policies that were put in place in Chile to face the COVID-19 pandemic within a liberal welfare framework. Social protection policies have been strongly stressed by the impact of the COVID-19 pandemic, which have had to face serious problems of unemployment and decreased family income. The nature of the measures arranged for the emergency situation depends on the welfare structure that the countries have built. Chile is a paradigmatic case of a neoliberal regime. Decisions to combat socio-economic impacts have maintained a subsidiary character with a strong predominance of the private sector in tandem with targeted coverage where the State's attention has been focused especially on the most vulnerable population. The characteristics of the distribution of social benefits have not achieved higher levels of equity and social cohesion but, on the contrary, they are moving away from the possibility of providing universal benefits by not incorporating redistributive and solidarity components in social security. The continuity in the neoliberal welfare structure leads us to propose that social policies, especially those linked to social protection, have widened the great inequality gaps in the population. 
Keywords: COVID-19; Social Policy; Social Protection; Neoliberalism; Inequality.

$1 \quad$ Introducción: Pandemia y políticas de protección social

Tras más de un año de pandemia del COVID-19, el impacto que ha significado su propagación no sólo se expresa en la pérdida de miles de vidas, sino que también se ha transformado en una amenaza a la situación socioeconómica de amplios grupos de la población. El aumento de personas en situación de pobreza, la pérdida de empleos, la sobrecarga de trabajo de las mujeres, los problemas de salud mental, el hambre, entre otros, han generado un aumento en las brechas de inequidad para enfrentar la pandemia, que interpela a los gobiernos a tomar medidas y a desplegar acciones para gestionar la respuesta a la crisis desatada.

Los distintos arreglos de política pública de protección social realizados para enfrentar la crítica situación provocada por el COVID-19, respondieron, por una parte, a la emulación de lo realizado por los países donde la pandemia había llegado antes y a las recomendaciones de los organismos internacionales, especialmente de la OMS, pero en concordancia con la estructura de bienestar que han desarrollado los países en las últimas décadas, la que está determinada por las características en que se articula la triada familia, mercado y Estado (ESPING-ANDERSEN, 2000). La interacción, rol y peso de cada uno de estos actores definen el régimen de bienestar dispuesto para enfrentar los riesgos que afectan a las personas, en este caso, los riesgos derivados de la llegada de la pandemia.

En otras palabras, los procesos de desmercantilización y desfamiliarización que los Estados Ilevan a cabo a través de la oferta de 
políticas públicas definen las particularidades que adopta la distribución de los bienes y servicios al interior de una sociedad para hacer frente a las consecuencias de la llegada del virus. Por tanto, los regímenes de bienestar se despliegan no sólo en base a las acciones de protección social que desarrollan los Estados, sino también en base a las ofertas del mercado y de las estrategias que dispone el individuo, la familia y las instancias comunitarias (CECCHINI, 2019). Las crisis sociales, como la que ha afectado al mundo durante el 2020 y el 2021, no sólo representan la incapacidad de los Estados para proteger socialmente al conjunto de la población, sino también la fragilidad de la familia y las deficiencias del mercado, que no permiten que estas puedan suplir los beneficios que el Estado no ha sido capaz de entregar.

Aquellos países que han experimentado fuertes procesos de mercantilización de las políticas sociales en las décadas pasadas, han desplegado respuestas a los riesgos de la población en base al principio de la subsidiariedad del Estado: la focalización de la ayuda en los sectores más pobres y la individualización de la responsabilidad del bienestar, entre otras características de los regímenes de bienestar liberales (ESPING-ANDERSEN, 2000). El aumento de los índices de pobreza, la segmentación de las prestaciones sociales y la exclusión de sectores de la población son las expresiones de las deficiencias de los sistemas de protección social liberal frente a la emergencia actual (CEPAL, 2021). En síntesis, la crisis provocada por la pandemia demuestra que los Estados liberales que no logren generar modificaciones significativas para dar respuestas a los deficientes procesos de desmercantilización y desfamiliarización de la población, hoy afectada por la propagación del virus, profundizarán las diferencias entre grupos más privilegiados y otros menos favorecidos, en función de la capacidad de pago en el mercado o acceso a redes familiares o comunitarias que estos tengan para enfrentar el impacto de la crisis. En el siguiente capítulo, se revisarán las principales políticas sociales liberales llevadas a cabo en el país, luego se presentará la metodología de análisis secundarios de la encuesta CASEN en Pandemia, y 
finalmente los resultados que apuntan a la discusión sobre porque en Chile se aplicaron políticas neoliberales para hacer frente a la pandemia

\section{$2 \quad$ Marco teórico}

2.1 Chile un caso paradigmático de protección social liberal

Los sistemas de protección social en Chile han sido ampliamente estudiados por diversas razones. Por una parte, debido a las profundas reformas sociales neoliberales que se llevaron a cabo precozmente a fines de la década de los 1970's del siglo XX que cambiaron radicalmente el régimen de bienestar del país, influenciando la adopción de políticas de mercado por el resto de América Latina al alero del Consenso de Washington. Actualmente, en la literatura sobre bienestar en Chile se constata que es un caso de estudio privilegiado debido a las reformas sociales realizadas por los gobiernos de centro izquierda a partir de la década del 2000 principalmente en materia de protección social, salud y pensiones. La discusión se ha centrado en la capacidad que tienen estas políticas para promocionar un tipo de bienestar más igualitario y universal rompiendo con la herencia neoliberal de las políticas sociales, posición que ha sido liderada por quienes sitúan estas reformas en lo que se ha denominado como Universalismo Básico (FILGUEIRA et al., 2005; HUBER; STEPHENS, 2012; PRIBBLE, 2013), en tanto, otros autores enfatizan en la perseverancia de un modelo de protección social con bases en las reformas de la década de los 1980 's y con reformas sectoriales que no han logrado impactar estructura neoliberal del reparto (CASTIGLIONI, 2012; FARIAS, 2019)

Las reformas neoliberales de la protección social fueron establecidas durante la década de los ochenta y consistieron en la privatización de las prestaciones sociales (tanto en las tareas de gestión como de ejecución de los programas sociales), la focalización de los beneficios en base a instrumentos de estratificación social, la disminución de los principios de 
solidaridad en el reparto, desregulación del mercado laboral, y prioridad en el crecimiento económico, aspectos que se han mantenido vigentes en el grueso de las prestaciones sociales hasta el día de hoy (MARTIN, 2016). Si bien, las reformas a la protección social llevadas a cabo durante la década del 2000 implicaron la ampliación de coberturas de programas y subsidios, así como la incorporación de la población informal a algunos de estos y el acceso garantizado a programas sociales específicos para ciertos sectores de la población estas no alteraron el modelo de políticas neoliberales (MARTIN, 2016). El mercado se ha mantenido como proveedor y administrador de las principales prestaciones sociales destinadas a las poblaciones de mayores recursos. En tanto el Estado ha mantenido su rol subsidiario, generando políticas de protección social hacia las poblaciones de menor capacidad de generación de ingresos. Concomitante a este fenómeno, se han desarrollado escasos procesos de desfamiliarización, no disminuyendo las dependencias familiares del bienestar, principalmente en lo referido a las tareas de cuidado infantil, de enfermos y adultos mayores a cargo de las mujeres (PALMA; SCOTT, 2020).

En resumen, en las últimas décadas el régimen de bienestar chileno ha mantenido los privilegios condicionados a la calidad contributiva de los beneficiarios y a la generación de ingresos. El carácter contributivo del sistema garantiza prioritariamente la protección a los asalariados con contrato de trabajo y a la tercera edad que recibe beneficios habida participación en el mercado laboral formal durante su vida productiva, al mismo tiempo que la capacidad de pago facilita el acceso a bienes y servicios sociales en el mercado. Sin embargo, la mayor flexibilidad y heterogeneidad del mercado laboral en los últimos años, ha marginado a sectores de trabajadores y a sus familias de las prestaciones sociales, a pesar de mantenerse en el mercado laboral, debido a la informalidad y bajos ingresos. Por su parte, los componentes no contributivos del sistema se han destinado preferentemente a la población en situación de pobreza bajo estrictos criterios de focalización - tales como los distintos subsidios habitacionales, educacionales, laborales destinados a los deciles más 
pobres. Tal población accede desventajosamente en término de calidad a las prestaciones sociales, profundizando la distancia del bienestar entre la población (FARÍAS, 2019).

De forma adicional a las transformaciones del mercado laboral, los cambios experimentados en las estructuras familiares han significado una fuerte presión para el cumplimiento de las labores de cuidado al interior de la familia y un desplazamiento de dichas funciones a las esferas del Estado y del mercado, generando complejos y débiles procesos de desfamiliarización (PALMA; SCOTT, 2020) en una sociedad conservadora y familiarista. La segmentación que presenta el régimen de bienestar chileno responde a pautas diversificadas históricamente para enfrentar los riesgos sociales. Se trata de un determinado arreglo político y social para la provisión del bienestar, que se transformó en una coyuntura crítica, desde la cual se instaló una trayectoria de desiguales posibilidades desmercantilizadoras y desfamilizadoras para la población.

Las políticas sociales han mantenido un carácter subsidiario con fuerte predominio del sector privado, con coberturas focalizadas donde la atención del Estado ha estado centrada especialmente en la población más vulnerable. Esto ha permitido que no se hayan logrado mayores niveles de equidad y cohesión social, sino que por el contrario se haya alejado la posibilidad de proveer prestaciones de carácter universal al no incorporar componentes redistributivos y solidarios en la seguridad social, "manteniéndose un sistema dual y altamente desigual en los servicios sociales, con transferencias asistenciales de bajo monto" (MARTIN, 2016, p.18).

La continuidad en la estructura de bienestar liberal, al no lograr alterar sustancialmente los niveles de mercantilización y familiarización así como tampoco la segmentación en la distribución de bienes y servicios entre la población, nos lleva a plantear que las políticas sociales, especialmente aquellas vinculadas a la protección social, se hayan visto fuertemente estresadas por la pandemia, al mismo tiempo que se hayan 
visualizado y ensanchado las grandes brechas de inequidad en la población.

\subsection{Intervenciones de protección social y estrategias privadas para enfrentar la pandemia}

Al inicio de la pandemia, Chile se encontraba en medio de una crisis política y social, que se había iniciado con movilizaciones masivas a partir del 18 de octubre del 2019. Las principales demandas de la ciudadanía fueron la necesidad de generar reformas estructurales tanto al sistema político como a las políticas sociales que permitieran mejorar la situación socioeconómica de la población y disminuir las amplias brechas de desigualdad presentes en el país, en un claro reclamo por modificar la estructura de bienestar liberal que ha marcado las últimas décadas de las políticas sociales en Chile.

Las políticas de protección social son una herramienta indispensable para enfrentar los efectos negativos que se han derivado de la pandemia y procurar alivio socioeconómico a las poblaciones afectadas. Para el caso chileno, una vez arribado el virus al país, el gobierno del presidente Sebastián Piñera presentó en marzo de 2020 un Plan de Emergencia Económica que comprometía una inversión de US\$11.750 millones para apoyar a las empresas y hogaresfrente al impacto negativo de la pandemia. Los ejes principales del plan fueron: a) reforzar el presupuesto del sistema de salud a cargo del $2 \%$ constitucional para atender los gastos que se deriven de la emergencia sanitaria; b) un eje para asegurar los ingresos de los trabajadores, a través del acceso a recursos propios de los seguros de cesantía por parte de los trabajadores contributivos; y la entrega de una transferencia monetaria (Bono Covid 19) vía focalización en los hogares de menores ingresos y a trabajadores informales, el cual estaba destinado a 2 millones de personas con un gasto de US\$130 millones de dólares, y c) el tercer eje, orientado a las pequeñas y medianas empresas, 
consideraba medidas tributarias, entre otras postergaciones del pago de impuestos tales como pagos provisionales mensuales, impuesto a la renta de empresas y contribuciones, entre otras (GOBIERNO DE CHILE, 2020) .

Las medidas anunciadas para los trabajadores se enmarcaron en la denominada Ley de Protección al Empleo (ley 21.227 de 6 de abril de 2020) destinada a los trabajadores regidos por el Código del Trabajo y que estén afiliados al seguro de cesantía, la cual consideró la suspensión temporal de los efectos y obligaciones del contrato de trabajo; es decir, el trabajador no queda obligado a prestar servicios y el empleador no estará obligado al pago de la remuneración respectiva. Esta estrategia fue fuertemente criticada por sectores de la oposición al gobierno, principalmente por el uso de los dineros del seguro de cesantía para suplir los ingresos de las y los trabajadores que por motivos de la emergencia sanitaria tuviesen que permanecer en sus domicilios sin posibilidad de realizar las labores a distancia. Esta decisión más que una política de protección social es un préstamo individual, que proviene de las cuentas de los trabajadores formales, con acuerdo del empleador, que se vieron obligados por la crisis a usar sus fondos para financiar sus salarios. Esta disposición legal además dejaba fuera de la estrategia a las y los trabajadores informales no contributivos que no cuentan con estos recursos. De acuerdo con datos de la Superintendencia de Pensiones, durante el 2020 hubo 5.977 .593 afiliados activos, es decir, que presentan algún tipo de cotización, no obstante, la fuerza de trabajo es de 7.990 .000 personas (SUPERINTENDENCIA DE PENSIONES, 2021).

Posteriormente, en el mes de mayo de 2020 se implementó una política de protección social que incluía el Ingreso Familiar de Emergencia (IFE Covid) y el Bono Covid, (Ley $\left.n^{\circ} 21.230\right)$, la que consistía en transferencias monetarias para los hogares de menores ingresos con trabajadores informales que vieron disminuidos sus ingresos debido a la emergencia sanitaria (BCN, 2020). La distribución del beneficio se relacionaba con el tipo de cuarentena en que se encontraba la comuna de residencia de los 
beneficiarios y el monto del beneficio se estableció según de la cantidad de personas que integraran el hogar y la situación socioeconómica de la familia, de acuerdo con criterios de focalización y a procesos de selección basados en el Registro Social de Hogares (CHILE ATIENDE, 2020).

Los beneficios sociales fueron establecidos inicialmente por un periodo de 4 meses, los que han sido prorrogados en los meses siguientes, variando algunos de los requisitos, así como los montos de las transferencias debido a la extensión de la pandemia, manteniéndose bajo criterios de focalización en los sectores más vulnerables e informales. A partir del mes de abril de 2021, se anunciaron cambios en el IFE Covid y Bono Covid, aumentando los montos de los beneficios, ampliando la cobertura, y simplificando el acceso (en el cual ya no se consideró el tipo de cuarentena del territorio). La última extensión fue realizada el 26 de mayo de 2021, aumentando los montos del IFE al nivel de la línea de la pobreza, luego de fuertes presiones de los diputados y senadores de los partidos de oposición y por los resultados electorales que castigaron duramente al gobierno en las elecciones del 15 y 16 de mayo de 2021.

La entrega de subsidios y transferencias monetarias (bonos) han significado una política de continuidad con el carácter subsidiario de la protección social característicos de los gobiernos de centroderecha que ponen énfasis en subsidios y bonos focalizados en los más pobres. Sin embargo, la magnitud de la crisis socioeconómica derivada de la emergencia sanitaria afectó duramente a la denominada clase media producto del impacto de las medidas de aislamiento y reducción de la movilidad, no siendo considerada esta población inicialmente en las medidas de protección social de los primeros meses de la pandemia, y entrando como beneficiarios recién el año 2021.

En abril de 2021, el gobierno generó un beneficio de aporte fiscal para la clase media consistente en una transferencia de hasta 500 mil pesos $^{3}$, no reembolsables, que se entregaron por única vez a trabajadores y empresarios individuales, que hayan tenido una disminución de, al menos,

\footnotetext{
${ }^{3}$ Equivalente a 690 dólares según cambio al 27 de mayo de 2021.
} 
el 20\% de sus ingresos como consecuencia de la emergencia sanitaria y focalizado en aquellos que el promedio de sus ingresos formales sea mayor al salario mínimo y menor a \$2.000.0004. Junto a ello se entregó un préstamo clase media, para quienes producto de la emergencia sanitaria, enfrentaron una disminución superior al 10\% de sus ingresos formales, que se puede devolver en cuatro cuotas anuales y sucesivas, sin multas ni intereses. El préstamo permite cubrir hasta el 100\% de la caída de los ingresos, con un tope de $\$ 650.000^{5}$ mensuales, que puede solicitarse hasta en tres oportunidades. (MINISTERIO DE DESARROLLO SOCIAL Y FAMILIA, 2021)

Las medidas de protección social enunciadas en los párrafos anteriores han sido insuficientes para aminorar el impacto de la caída de los ingresos de la población luego de más de un año de restricciones al trabajo y la movilidad, lo que se ha expresado entre otros aspectos en aumento de la pobreza, de los asentamientos informales que aumentaron en 74\% desde el 2019 (CHILE TECHO, 2021) y el escaso cumplimiento de las cuarentenas decretadas en los diferentes territorios del país debido a que gran cantidad de personas salen a trabajar por la falta de recursos. Por ello, una de las propuestas más debatidas durante el 2020 y 2021, ha sido la posibilidad de que las y los trabajadores contribuyentes puedan retirar el 10\% de los fondos previsionales de sus cuentas de capitalización individual, los cuales son administrados por las administradoras de pensiones AFP. Esta iniciativa surge desde el Congreso por parte de los partidos políticos contrarios al gobierno en respuesta a la escasa ayuda que el gobierno ha entregado a la ciudadanía y se enfrentó a una fuerte oposición por parte del ejecutivo en las tres ocasiones que se ha presentado tramitado. El 30 de julio de 2020, se aprobó el primer retiro del 10\%, al cual continuaron dos retiros más por el mismo porcentaje, el segundo el 10 de diciembre de 2020 y el tercero el 28 de abril de $2021^{6}$.

\footnotetext{
${ }^{4}$ Para ingresos mayores a 450 y menores a 2.760 dólares de mayo de 2021.

${ }^{5}$ Equivalente a 897 dólares según cambio al 27 de mayo de 2021.

${ }^{6}$ Leyes 21.248; 21.295; y 21.330 respectivamente.
} 
A marzo del 2021, la Superintendencia de Pensiones, organismo regulador del gobierno en materia de pensiones, informaba que luego del segundo retiro 7.524 .023 personas afiliadas al sistema de AFPs habían retirado sus fondos por un monto total accediendo a un total de U\$34.995 millones, dejando sin recursos en sus fondos de pensiones a 2.953 .525 personas (SUPERINTENDENCIA DE PENSIONES, 2021). Datos que demuestran la urgente necesidad de ingresos monetarios de gran parte de la población para enfrentar la pandemia, y como la estrategia desarrollada apeló a los recursos individuales de los trabajadores debilitando los fondos ahorrados para su vejez.

Las medidas de acceso a los fondos individuales por parte de las y los trabajadores contribuyentes, tanto de los recursos del fondo de cesantía, como de los ahorros previsionales en las administradoras de pensiones privadas, dan cuenta de intervenciones neoliberales que privilegian las estrategias de mercado por sobre políticas sociales más solidarias y redistributivas, y al mismo tiempo instalan la responsabilidad del bienestar en el individuo y en los recursos que este puede agenciar en base a su incorporación al mercado laboral y sus propias redes familiares y comunitarias, continuando con una trayectoria establecida décadas antes.

Por otra parte, las políticas de protección social han mantenido a su vez una lógica de focalización y subsidiariedad, propias de los regímenes de bienestar liberales y han traído como consecuencia mayor inequidad en el reparto de los beneficios, aumentando no solo los indicadores de pobreza sino también ampliando aún más les brechas de desigualdad existentes en el país, demostrando coherencia entre la política emprendida por el gobierno de Sebastián Piñera y los principios neoliberales de la protección social. 
En tiempos de pandemia COVID-19, el Ministerio de Desarrollo Social y Familia -ente de gobierno encargado de focalizar las ayudas sociales- ha desarrollado la Encuesta Social COVID-19 cuyo foco se centra en las consecuencias sociales y económicas que la pandemia ha tenido en los hogares del país.

El objetivo de la Encuesta fue conocer la situación de ingresos de las familias a partir de los cambios en la situación de trabajo asociados a la pandemia COVID-19 y su impacto en el acceso a bienes de consumo y servicios esenciales para la familia, como salud y educación.

La Encuesta7 se aplicó en dos olas, julio y noviembre del 2020, y tiene variables individuales, de hogares y territoriales. El marco muestral de la ola 1 considera a hogares encuestados en la Encuesta Nacional de Empleo (ENE) en los años 2017 y 2018 y usó un diseño bifásico a partir de las viviendas levantadas en la ENE cuyo diseño muestral es probabilístico, estratificado y bietápico. La Encuesta cuenta con una representatividad nacional y a nivel de macrozonas: Macrozona Norte (Arica y Parinacota, Tarapacá, Antofagasta, Atacama, Coquimbo); Macrozona Centro (Valparaíso, O'Higgins, Maule); Macrozona Sur (Ñuble, Biobío, Araucanía, Los Ríos, Los Lagos); Macrozona Extremo Sur (Aysén y Magallanes) y Macrozona Metropolitana (Región Metropolitana). Posee un error de 2,7\% a nivel nacional y de $4,7 \%$ a nivel macrozonal suponiendo máxima heterogeneidad. Su aplicación fue telefónica a habitantes de los hogares mayores de 18 años. En total se consideraron: 4.387 viviendas, 4.426 hogares y 13.648 personas en hogares.

La segunda ola se realizó en noviembre del 2020 y contempló un diseño muestral similar al anterior, pero llegó a una muestra efectiva de: 3.323 
viviendas, 3.333 hogares y 10.344 personas en hogares. Esto implicó un error máximo en macrozona de 4,7\%.

Para efectos de este estudio, se utilizó la base de datos de ambas olas, generando un análisis ad-la

hoc a partir de ellos. La estrategia de datos utilizadas fueron principalmente datos descriptivos de la situación chilena en pandemia.

\section{$4 \quad$ Resultados}

4.1 Chile y COVID: La neoliberalización de la asistencia estatal

La pandemia COVID 19 ha profundizado las desigualdades existentes entre los hogares más ricos y los más pobres, llevando a situaciones de extrema pobreza y exclusión social que no se veían en el país desde la profunda crisis económica de la década de los 80 , producto de la implementación de la transformación neoliberal del sistema impuesto por la dictadura militar. En el Gráfico 1, se muestra la evolución de la tasa de la pobreza desde la década de los '90

Gráfico 1: Incidencia de la pobreza por ingresos

(Metodología actual)

$\%$ personas

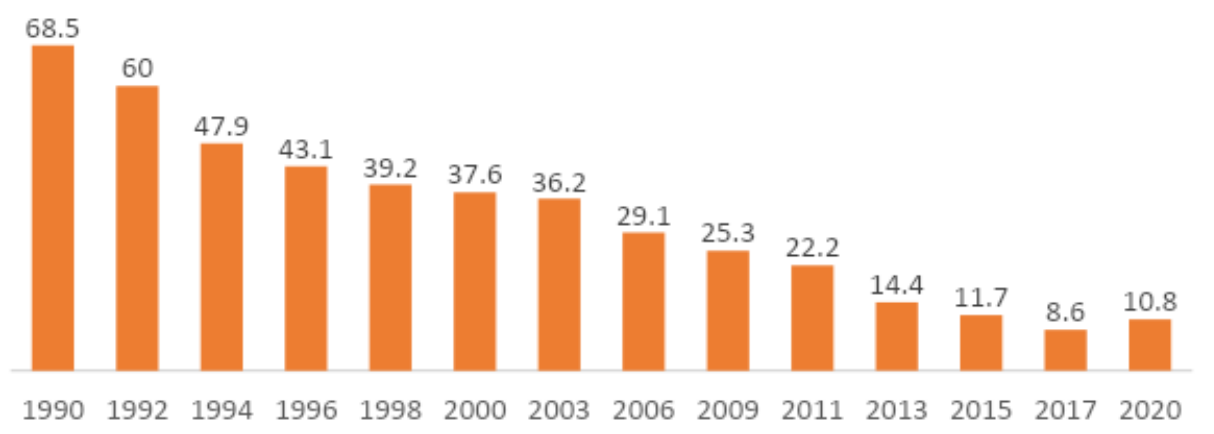

Fuente: Casen 2020 (MINISTERIO DEL DESARROLLO SOCIAL Y FAMILIA, 2020).

Según datos de la encuesta COVID-19 del Ministerio de Desarrollo Social y Familia se estima que la pobreza por ingresos ha aumentado en 4,1 
puntos porcentuales lo que equivale a 800.000 familias. Esta condición se da principalmente por la pérdida de empleo de los hogares, en donde el 36,9\% de los trabajadores activos ha visto trastocada su situación laboral a través de despidos o suspensiones de contratos permitidas por el estado para proteger a las empresas del costo del COVID-19. De acuerdo con los datos de la encuesta, el 52,7\% del quintil 1 de ingresos ha perdido o visto suspendido su empleo en comparación al 11,5\% del quintil 5. Las imposibilidades de trabajo no solo se deben a la suspensión de empresas, sino a la ejecución del mandato de "Quédate en casa", que ha sido la principal consigna usada por el gobierno para hacer frente a la pandemia. Sin embargo, el 35,7\% del quintil 1 son trabajadores no calificados y un 19,7\% se desempeña en actividades de servicios o comercios (MINISTERIO DEL DESARROLLO SOCIAL Y FAMILIA, 2017), por ende quedarse en casa es no llevar ingresos al hogar.

El impacto de la pérdida de empleo se refleja directamente en la pérdida de ingresos, el 62,7\% de los hogares del primer quintil han visto disminuidos sus ingresos comparados con el 24,9\% del quinto quintil (ola 1). El 45.6\% de los hogares declara que la pérdida de ingresos llega a más de la mitad de lo que percibían antes de la pandemia, pero esto se profundiza en el primer quintil que alcanza el 55,6\% en comparación al quinto quintil en donde el $30,6 \%$ ha visto disminuidos sus ingresos en más de la mitad. Esta pérdida de ingresos es muy relevante, si se considera que antes de la pandemia el $77 \%$ de los hogares del primer quintil consideraba que los ingresos que tenía les alcanzaba para vivir.

En este escenario, un 57,5\% ha recibido ayuda, siendo la estatal la principal con un 79,6\%. Dentro de las medidas implementadas por el Estado el Ingreso Familiar de Emergencia (IFE) ha sido recibido por un $37,8 \%$ de las familias, el bono de clase media por un 10,8\% y el crédito de clase media por un 5,7\%. Claramente estas medidas han sido insuficientes, dado que como se aprecia en la Tabla 1, se han debido utilizar ahorros del hogar, principalmente en los dos primeros quintiles de ingreso que no 
tienen muchas redes para pedir dinero o vender enseres. Lo que queda de manifiesto con estos datos, es que las acciones emprendidas han sido principalmente gestionadas en forma individual en cada hogar.

Tabla 1: Acciones realizadas para tener dinero durante la pandemia COVID-19.

\begin{tabular}{|c|c|c|c|c|c|c|}
\hline & Quintil I & Quintil II & Quintil III & Quintil IV & Quintil V & Total \\
\hline Retirar el $10 \%$ de la AFP & $73,3 \%$ & $83,9 \%$ & $82,0 \%$ & $86,6 \%$ & $73,6 \%$ & $79,6 \%$ \\
\hline $\begin{array}{l}\text { Reducir los gastos en } \\
\text { alimentación }\end{array}$ & $54,2 \%$ & $44,4 \%$ & $42,5 \%$ & $33,2 \%$ & $23,5 \%$ & $41,4 \%$ \\
\hline $\begin{array}{l}\text { Reducir los gastos en } \\
\text { salud, incluir salud mental } \\
\text { y dental }\end{array}$ & $42,2 \%$ & $42,4 \%$ & $37,7 \%$ & $25,7 \%$ & $23,9 \%$ & $35,7 \%$ \\
\hline No pagar otras deudas & $27,4 \%$ & $24,6 \%$ & $20,3 \%$ & $17,1 \%$ & $10,2 \%$ & $20,9 \%$ \\
\hline No pagar agua, gas o luz & $32,6 \%$ & $22,5 \%$ & $18,1 \%$ & $13,9 \%$ & $9,7 \%$ & $20,7 \%$ \\
\hline $\begin{array}{l}\text { No pagar teléfono, } \\
\text { internet, u otros servicios } \\
\text { de comunicación }\end{array}$ & $30,1 \%$ & $20,5 \%$ & $17,3 \%$ & $17,0 \%$ & $7,1 \%$ & $19,5 \%$ \\
\hline Renegociar otras deudas & $20,2 \%$ & $22,4 \%$ & $20,1 \%$ & $18,1 \%$ & $11,3 \%$ & $18,9 \%$ \\
\hline $\begin{array}{l}\text { Reducir los gastos o dejar } \\
\text { de pagar calefacción, } \\
\text { parafina, leña }\end{array}$ & $19,5 \%$ & $18,0 \%$ & $14,8 \%$ & $12,5 \%$ & $9,5 \%$ & $15,4 \%$ \\
\hline Pagar menos arriendo & $4,7 \%$ & $7,2 \%$ & $3,5 \%$ & $9,1 \%$ & $2,1 \%$ & $5,3 \%$ \\
\hline No pagar el arriendo & $6,7 \%$ & $3,4 \%$ & $2,6 \%$ & $5,4 \%$ & $1,2 \%$ & $4,0 \%$ \\
\hline Renegociar el dividendo & $1,2 \%$ & $5,3 \%$ & $2,1 \%$ & $3,2 \%$ & $8,4 \%$ & $3,8 \%$ \\
\hline No pagar dividendo & $2,4 \%$ & $2,3 \%$ & $2,3 \%$ & $1,9 \%$ & $4,2 \%$ & $2,6 \%$ \\
\hline $\begin{array}{l}\text { Cambiarse de casa o } \\
\text { departamento }\end{array}$ & $1,9 \%$ & $3,1 \%$ & $2,8 \%$ & $2,6 \%$ & $1,7 \%$ & $2,4 \%$ \\
\hline No pagar gastos comunes & $2,6 \%$ & $2,5 \%$ & $1,0 \%$ &, $7 \%$ &, $7 \%$ & $1,6 \%$ \\
\hline
\end{tabular}

Fuente: Elaboración propia en base a Encuesta Social COVID-19 ola 2 (noviembre 2020).

Estas acciones han apelado al uso de ahorros individuales, la reducción de gastos en alimentación o salud y el cese del pago de servicios básicos, sobre todo en el contexto que el gobierno ha dictaminado la imposibilidad de las empresas de suspender los servicios básicos en pandemia, a pesar de las deudas (BCN, 2021).

Otro elemento relevante de la pandemia ha sido el hambre, que se ha traducido en que el 53,1\% de los hogares del quintil 1 se hayan preocupado por no tener que comer, el 47,5\% haya comido menos de lo que tenía planificado por falta de dinero u otros recursos, el 46,1\% comió poca variedad de alimentos o que el $41,5 \%$ no haya accedido a alimentos saludables. Reflejo de esta situación es que figuras que habían quedado 
enterradas desde la dictadura militar, como las "ollas comunes" han vuelto a aparecer en las organizaciones vecinales, para resolver de manera colectiva los problemas de falta de alimentación.

\subsection{Gestión de la Pandemia: Traspaso de responsabilidades estatales a la familia}

Desde el año 2003, se establece la Ley de Enseñanza Obligatoria de 12 años que debe ser proveída de forma gratuita por el Estado. En el contexto de pandemia, el 12 de marzo del 2020 se decretó el cierre de los colegios y la implementación de un sistema de educación a distancia que supone la instalación de clases sincrónicas o asincrónicas dependiendo de la realidad de cada establecimiento educacional. En la práctica la cantidad de actividades recibidas por los niños, como se ve en la Tabla 2, ha sido menor en los quintiles de menos ingresos.

Tabla 2: Tipo de actividades escolares recibida por el niño o niña en la última semana

\begin{tabular}{|l|r|r|r|r|r|r|}
\hline & Quintil I & Quintil II & Quintil III & Quintil IV & Quintil V & \multicolumn{1}{l|}{ Total } \\
\hline $\begin{array}{l}\text { Clases virtuales con } \\
\text { interacción con el } \\
\text { profesor }\end{array}$ & $76,9 \%$ & $83,0 \%$ & $84,5 \%$ & $87,2 \%$ & $87,8 \%$ & $82,7 \%$ \\
\hline $\begin{array}{l}\text { Guías de ejercicio y/0 } \\
\text { tareas por internet }\end{array}$ & $72,0 \%$ & $79,1 \%$ & $73,7 \%$ & $77,4 \%$ & $77,3 \%$ & $75,4 \%$ \\
\hline $\begin{array}{l}\text { Guías de ejercicio y/0 } \\
\text { tareas impresas }\end{array}$ & $60,8 \%$ & $50,6 \%$ & $59,7 \%$ & $54,4 \%$ & $49,6 \%$ & $56,0 \%$ \\
\hline $\begin{array}{l}\text { Clases virtuales sin } \\
\text { interacción con el } \\
\text { profesor }\end{array}$ & $23,3 \%$ & $23,2 \%$ & $22,1 \%$ & $27,1 \%$ & $19,0 \%$ & $23,1 \%$ \\
\hline $\begin{array}{l}\text { Clases presenciales } \\
\text { Clases por televisión }\end{array}$ & $4,3 \%$ & $4,7 \%$ & $3,6 \%$ & $8,8 \%$ & $2,1 \%$ & $4,7 \%$ \\
\hline
\end{tabular}

Fuente: Elaboración propia en base a encuesta Social COVID-19 ola 2 (noviembre 2020).

Uno de los elementos que explica esta brecha es que el 8\% de los alumnos no tiene conexión a internet para hacer educación a distancia, cifra que se incrementa en $15,3 \%$ en el quintil 1. En términos de equipamiento, el $84,1 \%$ de los hogares declara tener un computador o 
equipamiento para hacer clases, sin embargo, esta cifra alcanza sólo el $77,5 \%$ en los hogares del quintil 1.

La educación a distancia no sólo ha sido un desafío para docentes y estudiantes, sino que también para los apoderados, que en promedio declaran disponer de una hora para acompañar a sus hijos, pero el 42,7\% de los hogares declara que ha tenido dificultades para apoyarlos en este proceso escolar.

Por otra parte, las familias han tenido que tomar decisiones asociadas a la continuidad de estudios de sus hijos, ya sea por la imposibilidad de acompañamiento o imposibilidad de pago ${ }^{8}$, donde las principales postergaciones se han producido en educación básica-media y en educación superior, tal como se muestra en la Tabla 3.

Tabla 3: Decisiones familiares respecto a continuidad de estudios.

\begin{tabular}{|c|c|c|c|c|c|c|}
\hline & Quintil I & Quintil II & Quintil III & Quintil IV & Quintil V & Total \\
\hline $\begin{array}{l}\text { Dejar de pagar o retirar a } \\
\text { estudiantes de jardín } \\
\text { infantil o educación } \\
\text { preescolar }\end{array}$ & $27,2 \%$ & $27,8 \%$ & $32,0 \%$ & $22,8 \%$ & $44,9 \%$ & $28,0 \%$ \\
\hline $\begin{array}{l}\text { Dejar de pagar o retirar a } \\
\text { estudiantes de educación } \\
\text { básica, media o educación } \\
\text { especial }\end{array}$ & $32,6 \%$ & $33,4 \%$ & $29,6 \%$ & $38,1 \%$ & $6,3 \%$ & $32,0 \%$ \\
\hline $\begin{array}{l}\text { Cambiar a algún niño o niña } \\
\text { a una escuela o colegio más } \\
\text { económico }\end{array}$ & $8,1 \%$ & $19,4 \%$ & $1,4 \%$ &, $6 \%$ & $13,6 \%$ & $8,5 \%$ \\
\hline $\begin{array}{l}\text { Dejar de pagar, congelar o } \\
\text { aplazar los estudios en } \\
\text { educación superior }\end{array}$ & $43,0 \%$ & $31,5 \%$ & $31,5 \%$ & $23,5 \%$ & $21,7 \%$ & $37,1 \%$ \\
\hline
\end{tabular}

Fuente: Elaboración propia en base a encuesta Social COVID-19 ola 1- (julio 2020).

También la salud se ha visto afectada. En un país donde ya existe una extensa lista de espera en atenciones de salud, la pandemia ha profundizado esta crisis: la postergación de las médicas o dentales ha afectado al $83 \%$ de los hogares, el $62 \%$ ha postergado exámenes médicos, el $56,6 \%$ los tratamientos médicos y un $53,8 \%$ la compra de medicamentos.

\footnotetext{
${ }^{8}$ En Chile el 54\% de la matrícula está en los colegios Particulares Subvencionados que exige un copago de las familias para el acceso a educación (Cooperativa, 2021);
} 


\section{Conclusiones}

El modelo neoliberal y subsidiario que posee el Estado chileno se ha visto sobrepasado por la pandemia de coronavirus, en donde las demandas por cuidado y bienestar no solo provienen de los sectores de menores ingresos sino también por parte de los grupos medios.

La vulnerabilidad de los sectores de bajos ingresos, así como de los ingresos medios, se ha visto acrecentada de una forma sin precedentes en el contexto de la pandemia, lo que ha presionado a que en el marco de un sistema subsidiario la principal vía de hacer frente a la pandemia sea mediante el uso de instrumentos de ahorro individual, como ha sido el retiro del $10 \%$ de los fondos de pensiones individuales o los recursos de los seguros de cesantía, o de gestiones de recursos a través de las rede propias durante la pandemia.

La fragilidad de las políticas de protección social también ha repercutido a las otras esferas de intervención estatal, como son las provisiones de servicios de salud y educación que además de apelar a la gestión en base a los ingresos familiares, también ha introducido el uso de recursos humanos y de cuidado familiar que ha implicado una sobrecarga en las funciones familiares del cual no se tiene precedente.

En este contexto, el futuro presentará enormes desafíos en la construcción de mecanismos que permitan hacer frente a estas pérdidas de ingresos, recursos y prestaciones públicas en pos de velar por la protección de hogares de sectores medios que han quedado enormemente vulnerables frente a la pandemia, al mismo tiempo que el Estado deberá generar mecanismos que cierren las brechas de desigualdad que el COVID-19 ha dejado sobre las familias. 
BCN (Biblioteca del Congreso Nacional de Chile). Ley 21230, de 3 de agosto de 2020. Concede un ingreso familiar de emergencia. Diario Oficial 3 ago. 2020. BCN: Chile.

Disponible

en: https://www.bcn.cl/leychile/navegar?idNorma=1145400\&idParte=10121381\&idVersion =2020-08-03. Accedido en: 26 oct. 2021.

BCN (Biblioteca del Congreso Nacional de Chile). Suspensión del corte de servicios básicos de los clientes deudores. BCN: Chile. 24 mayo 2021. Disponible en:www.bcn.cl/leyfacil/recurso/suspension-del-corte-de-servicios-basicos-de-los-cli entes-deudores. Accedido en: 26 oct. 2021

CASTIGLIONI, Rossana. Social Policy Reform and Continuity under the Bachelet Administration. In: DÍEZ, Jordi; FRANCESCHET, Susan. Comparative Policy in Latin America, 247-271. Toronto: University of Toronto Press, 2012. https://doi.org/10.3138/9781442689947-012.

CECCHINI, Simone Protección social universal en América Latina y el Caribe. CEPAL: Naciones Unidas, 2019.

CEPAL (Comisión Económica para América Latina y el Caribe), Revista CEPAL, N¹32 (LC/PUB.2021/4-P), Santiago, 2021.

CHILE ATIENDE. Ingreso Familiar de Emergencia (IFE). Chile Atiende. 24 mayo 2020. Disponible en: https://www.chileatiende.gob.cl/fichas/78385-ingreso-familiar-de-emergencia.

Accedido en: 26 out. 2021

CHILE, Techo. Catastro de Campamentos 2020-2021. Santiago: Centro de Estudios Sociales Techo Fundación Vivienda, 2021.

COOPERATIVA [Cooperativa.CL]. Matrículas en la educación pública consolidan aumento y crecen en más de 11 mil respecto a 2018. Cooperativa.CL, Santiago. 16 ago. 2019.2 Disponible en: https://www.cooperativa.cl/noticias/pais/educacion/colegios/matriculas-en-la-educ acion-publica-consolidan-aumento-y-crecen-en-mas-de/2019-08-16/014902.html\#: : :text=Seg\%C3\%BAn\%2Olos\%20datos\%20del\%20Mineduc,Ministerio\%20de\%20Edu caci\%C3\%B3n\%20(Mineduc). Accedido en: 16 ago. 2019.

ESPING-ANDERSEN, Gosta. Fundamentos Sociales de las economías post industriales. Barcelona: Ariel, 2000.

FARÍAS, Ana. Políticas sociales en Chile: Trayectoria de inequidades y desigualdades en distribución de bienes y servicios. Santiago: Ed. Universidad Alberto Hurtado, 2019.

FILGUEIRA, Fernando et al. Universalismo básico: una propuesta posible y necesaria para mejorar las condiciones de vida. In: MOLINA, Gerardo. Universalismo básico: una nueva política social para América Latina. 19-57. Washington D. C.: Banque Interaméricaine de Développement, 2006. 
GOBIERNO DE CHILE. Presidente Piñera anunció un Plan de Emergencia Económica para proteger los ingresos laborales, el empleo y las PyMes. 19 mar. 2020. Disponible en:

https://www.gob.cl/noticias/presidente-pinera-anuncio-un-plan-de-emergencia-ec onomica-para-proteger-los-ingresos-laborales-el-empleo-y-las-pymes/. Accedido en: 26 out. 2021.

HUBER, Evelyne, STEPHENS, John.D. Democracy and the Left. Social Policy and inequality in Latin America. Chicago: The University of Chicago Press, 2012.

MARTíN, María Pía. Cambio o continuidad de las políticas de protección social en Chile en gobiernos de centroizquierda y centroderecha Revista CLAD, Reforma y Democracia. $N^{\circ} 64,2016: 193-222$.

MINISTERIO DE DESARROLLO SOCIAL Y FAMILIA. Encuesta Caracterización Socioeconómica Nacional (Casen). Observatorio social (Ministerio de Desarrollo Social y $\quad$ Familia). 2017.2 Disponible en: http://observatorio.ministeriodesarrollosocial.gob.cl/encuesta-casen-2017 Accesado en: 26 oct. 2021.

MINISTERIO DE DESARROLLO SOCIAL Y FAMILIA. Red de Protección Social. 28 de Mayo de 2021.2 Disponible en: https://www.reddeproteccion.cl/fichas/bono_clase_media_2021\#: :text=El\%20Bono \%20Clase\%20Media\%202021,.000\%20y\%20\%242.000.000 Accesado en: 26 oct. 2021.

PALMA, Julieta;; SCOTT, Jacqueline. The implications of changing living arrangements for intergenerational relations in Chile. Contemporary Social Science, 15(3), 2020: 392-405.

PRIBBLE, Jeniffer. Welfare and Party Politics in Latin America. Nueva York: Cambridge University Press, 2013. https://doi.org/10.1017/CBO9781139343299

SUPERINTENDENCIA DE PENSIONES. Superintendencia de Pensiones. 28 mayo $2021 . \quad$ Disponible en: ttps://www.spensiones.cl/portal/institucional/594/w3-article-14409.html. Accesado en: 28 mayo 2021. 